Volume 110 No. 1 2016, 1-7

ISSN: 1311-8080 (printed version); ISSN: 1314-3395 (on-line version)

url: http://www.ijpam.eu

doi: 10.12732 /ijpam.v110i1.1

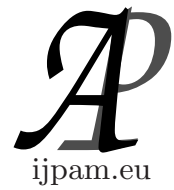

\title{
A NEW FAMILY OF SECANT-LIKE METHOD WITH SUPER-LINEAR CONVERGENCE
}

\author{
M. Imran ${ }^{1}$, Syamsudhuha ${ }^{2}$, Supriadi Putra ${ }^{3}$ \\ ${ }^{1,2,3}$ Numerical Computing Group \\ Department of Mathematics \\ University of Riau \\ Pekanbaru, 28293, INDONESIA
}

\begin{abstract}
Another new family of Secant-like method is proposed in this paper. Analysis of convergence shows that the method has a super-linear convergence as Secant method. Numerical experiments show that the efficiency of the method is depended on the value of its parameter.
\end{abstract}

AMS Subject Classification: 65D99, 65H05

Key Words: secant-like method, secant method, order of convergence, iterative method

\section{Introduction}

In this study, we are concerned of finding an approximated solution of a nonlinear equation of the form

$$
f(x)=0 .
$$

A classic known method without employing derivative to solve (1) is Secant method derived by passing a line through two point, says $\left(x_{0}, f\left(x_{0}\right)\right)$ and $\left(x_{1}, f\left(x_{1}\right)\right)$. The equation of this line is

$$
p(x)=f\left(x_{1}\right)+\frac{f\left(x_{1}\right)-f\left(x_{0}\right)}{x_{1}-x_{0}}\left(x-x_{1}\right) .
$$

The intersection of (2) with $x$-axis is the first approximation to the root of

Received: October 29, 2015

Revised: $\quad$ August 31, 2016

Published: October 21, 2016

$\S_{\text {Correspondence author }}$ (c) 2016 Academic Publications, Ltd.

url: www.acadpubl.eu 
(1). Repeating this proses, we obtain Secant iteration,

$$
x_{n+1}=x_{n}-f\left(x_{n}\right) \frac{x_{n}-x_{n-1}}{f\left(x_{n}\right)-f\left(x_{n-1}\right)}, \quad n \geq 1 .
$$

Secant method satisfies the equation error

$$
e_{n+1}=c_{2} e_{n-1} e_{n}
$$

where $c_{2}=\frac{f^{(2)}\left(x^{*}\right)}{2 ! f^{\prime}\left(x^{*}\right)}$ and its order is $(1+\sqrt{5}) / 2$ (super linear) [3]. Many works have been done developing new Secant-like methods, see $[1,2,4,5,6,7,8,9$, $10,11,12,13,14]$ for example.

Recently V. Kanwar, J.R. Sharma, and Mamta [10] introduces a variation of Secant method, derived by creating a parabola passing a point $\left(x_{1}, 0\right)$. The parabola's equation is

$$
y=\alpha\left(x-x_{1}\right)^{2},
$$

where $\alpha$ is a parameter. Parabola (5) intercepts $p(x)$ at $\left(x_{2}, p\left(x_{2}\right)\right)$, so that

$$
p\left(x_{2}\right)=f\left(x_{1}\right)+\frac{f\left(x_{1}\right)-f\left(x_{0}\right)}{x_{1}-x_{0}}\left(x_{2}-x_{1}\right),
$$

and

$$
p\left(x_{2}\right)=\alpha\left(x_{2}-x_{1}\right)^{2}
$$

From (6) and (7), we obtain

$$
\alpha\left(x_{2}-x_{1}\right)^{2}-\frac{f\left(x_{1}\right)-f\left(x_{0}\right)}{x_{1}-x_{0}}\left(x_{2}-x_{1}\right)-f\left(x_{1}\right)=0 .
$$

Solving the quadratic equation (8) yields

$$
x_{2}=x_{1}-\frac{2\left(x_{1}-x_{0}\right) f\left(x_{1}\right)}{f\left(x_{1}\right)-f\left(x_{0}\right) \pm \sqrt{\left(f\left(x_{1}\right)-f\left(x_{0}\right)\right)^{2}+4 \alpha f\left(x_{1}\right)\left(x_{1}-x_{0}\right)^{2}}} .
$$

Equation (9) suggests the iteration formula

$$
x_{n+1}=x_{n}-\frac{2\left(x_{n}-x_{n-1}\right) f\left(x_{n}\right)}{K+\operatorname{sign}(K) \sqrt{K^{2}+4 \alpha f\left(x_{n}\right)\left(x_{n}-x_{n-1}\right)^{2}}},
$$

where

$$
K=f\left(x_{n}\right)-f\left(x_{n-1}\right),
$$


$\alpha$ is a parameter and

$$
\operatorname{sign}(K)=\left\{\begin{array}{cl}
1 & , \text { untuk } K \geq 0 \\
-1 & , \text { untuk } K<0
\end{array}\right.
$$

for $n=1,2,3, \ldots$ Kanwar shows that the convergence of iterative method (10) is the same as Secant method.

Chen[4] rearranges (8) as

$$
\left(\alpha\left(x_{2}-x_{1}\right)-\frac{f\left(x_{1}\right)-f\left(x_{0}\right)}{x_{1}-x_{0}}\right)\left(x_{2}-x_{1}\right)-f\left(x_{1}\right)=0 .
$$

Equation (11) can be expressed as

$$
x_{2}-x_{1}=\frac{f\left(x_{1}\right)\left(x_{1}-x_{0}\right)}{\alpha\left(x_{2}-x_{1}\right)\left(x_{1}-x_{0}\right)-\left(f\left(x_{1}\right)-f\left(x_{0}\right)\right)} .
$$

By approximating $x_{2}$ in the right hand side of (12) with Secant method and simplifying, Chen obtains a super linearly convergent iterative method

$$
x_{n+1}=x_{n}-\frac{f\left(x_{n}\right)\left(x_{n}-x_{n-1}\right)\left(f\left(x_{n}\right)-f\left(x_{n-1}\right)\right)}{\left(f\left(x_{n}\right)-f\left(x_{n-1}\right)\right)^{2}+\alpha f\left(x_{n}\right)\left(x_{n}-x_{n-1}\right)^{2}} .
$$

In this article we discuss another variation of Secant method and its convergent analysis in Section 2. Then, in Section 3 we compare the proposed method with the methods given in (10) and (13) using some test functions.

\section{Proposed Method}

Dividing both side equation (8) with $\frac{f\left(x_{1}\right)-f\left(x_{0}\right)}{x_{1}-x_{0}}$ yields

$$
\alpha\left(x_{2}-x_{1}\right)^{2}\left(\frac{x_{1}-x_{0}}{f\left(x_{1}\right)-f\left(x_{0}\right)}\right)-\left(x_{2}-x_{1}\right)-f\left(x_{1}\right)\left(\frac{x_{1}-x_{0}}{f\left(x_{1}\right)-f\left(x_{0}\right)}\right)=0 .
$$

Rearranging the terms on equation (14), we obtain

$$
x_{2}-x_{1}=\alpha\left(x_{2}-x_{1}\right)^{2}\left(\frac{x_{1}-x_{0}}{f\left(x_{1}\right)-f\left(x_{0}\right)}\right)-f\left(x_{1}\right)\left(\frac{x_{1}-x_{0}}{f\left(x_{1}\right)-f\left(x_{0}\right)}\right) .
$$

By approximating $x_{2}$ in the right-hand side equation (15) with Secant method yields

$$
x_{2}=x_{1}-f\left(x_{1}\right)\left(\frac{x_{1}-x_{0}}{f\left(x_{1}\right)-f\left(x_{0}\right)}\right)+\alpha f^{2}\left(x_{1}\right)\left(\frac{x_{1}-x_{0}}{f\left(x_{1}\right)-f\left(x_{0}\right)}\right)^{3} .
$$


From (16), we propose the iterative formula

$$
x_{n+1}=x_{n}-f\left(x_{n}\right) L\left(1-\alpha f\left(x_{n}\right) L^{2}\right),
$$

where

$$
L=\frac{x_{n}-x_{n-1}}{f\left(x_{n}\right)-f\left(x_{n-1}\right)} .
$$

If we take $\alpha=0$, then equation (17) becomes Secant method.

Theorem 1. Assume that the function $f: D \subset \mathbb{R} \rightarrow \mathbb{R}$ for an open interval $D$ has a simple root $x^{*} \in D$ that is $f^{\prime}\left(x^{*}\right) \neq 0$. Let $f(x)$ have first, and second derivatives in the interval $D$, then the order of convergence of the method defined by $(17)$ is $(1+\sqrt{5}) / 2$.

Proof.Let $e_{n}=x_{n}-x^{*}$, then

$$
L=\frac{e_{n}-e_{n-1}}{f\left(x_{n}\right)-f\left(x_{n-1}\right)} .
$$

Taylor expansion of $f\left(x_{n}\right)$ about $x_{n}=x^{*}$ is given by

$$
f\left(x_{n}\right)=f^{\prime}\left(x^{*}\right)\left(e_{n}+c_{2} e_{n}^{2}\right)+\cdots,
$$

where $c_{j}=\frac{f^{(j)}\left(x^{*}\right)}{j ! f^{\prime}\left(x^{*}\right)}$. Similarly, we obtain

$$
f\left(x_{n-1}\right)=f^{\prime}\left(x^{*}\right)\left(e_{n-1}+c_{2} e_{n-1}^{2}\right)+\cdots .
$$

Subtracting (21) from (20) yields

$$
f\left(x_{n}\right)-f\left(x_{n-1}\right)=f^{\prime}\left(x^{*}\right)\left(\left(e_{n}-e_{n-1}\right)+c_{2}\left(e_{n}^{2}-e_{n-1}^{2}\right)+\cdots\right) .
$$

Since $e_{n}$ is small enough, then substituting (22) into (19) we obtain

$$
L=\frac{1}{f^{\prime}\left(x^{*}\right)\left(1+c_{2}\left(e_{n}+e_{n-1}\right)\right)} .
$$

Using geometry series, (23) can be written as

$$
L=\frac{1}{f^{\prime}\left(x^{*}\right)}\left(1-c_{2} e_{n}-c_{2} e_{n-1}+c_{2}^{2} e_{n}^{2}+2 c_{2}^{2} e_{n} e_{n-1}+c_{2}^{2} e_{n-1}^{2}\right) .
$$

By ignoring the terms containing $e_{n}^{j} e_{n}^{k}$, with $k+j>2$ yields

$$
L^{2}=\frac{1}{\left(f^{\prime}\left(x^{*}\right)\right)^{2}}\left(1+6 c_{2}^{2} e_{n} e_{n-1}-2 c_{2} e_{n}-2 c_{2} e_{n-1}+3 c_{2}^{2} e_{n}^{2}+3 c_{2}^{2} e_{n-1}^{2}\right) .
$$


Then, using (21), (24), dan (25) we have

$$
f\left(x_{n}\right) L\left(1-\alpha f\left(x_{n}\right) L^{2}\right)=e_{n}-c_{2} e_{n} e_{n-1}-\alpha \frac{e_{n}^{2}}{f^{\prime}\left(x^{*}\right)} .
$$

On substituting (26) into (17), and recalling $e_{n+1}=x_{n+1}-x^{*}$, we get

$$
e_{n+1}=c_{2} e_{n-1} e_{n}+\alpha \frac{e_{n}^{2}}{f^{\prime}\left(x^{*}\right)} .
$$

Equation (27) is the same as equation (4), so the order of convergence the iterative method $(17)$ is $(1+\sqrt{5}) / 2$.

\section{Numerical Experiments}

In this section we compare the proposed method (17) with the methods introduced by Kanwar (10) and Chen (13) using the following test functions

$$
\begin{array}{llrl}
f_{1}(x) & =\log (x), & x^{*} & =1.0, \\
f_{2}(x) & =x^{3}-2 x-5, & x^{*} & =2.094551481542327 \\
f_{3}(x) & =x \exp (x)-1, & x^{*} & =0.5671432904097839 . \\
f_{4}(x) & =x \exp (x)-\cos (x), & x^{*} & =0.5177573636824583 .
\end{array}
$$

We use different initial guesses and stop the iteration prosseses by the following condition

(a) Maximum number of iteration $=100$,

(b) If $\left|f\left(x_{n+1}\right)\right|<1.0 \times 10^{-14}$,

(c) If $\left|x_{n+1}-x_{n}\right|<1.0 \times 10^{-14}$.

The results are shown in Table 1 . In this table, NC indicates that the methods are not convergent or exceed the number iterations allowed or blow up.

From computational experiments, we can see that the proposed method (PM) is comparable with Kanwar's method (KM) and Chen's method (CM), especially for small enough $\alpha$. Hence, we can conclude that the proposed method can be used as an alternative method for Secant-like methods. 
Table 1: The number iterations needed to obtain the solution of $f(x)=$ 0 by varying parameter $\alpha$

\begin{tabular}{|c|c|c|c|c|c|c|c|c|c|c|}
\hline \multirow{2}{*}{ Function } & \multirow{2}{*}{$x_{0}$} & \multirow{2}{*}{$\mathrm{x}_{1}$} & \multirow{2}{*}{\multicolumn{2}{|c|}{ Method }} & \multicolumn{6}{|c|}{$\alpha$} \\
\hline & & & & & 10 & 1 & 0.1 & 0.01 & \multicolumn{2}{|c|}{0.001} \\
\hline \multirow{9}{*}{$\log (\mathbf{x})$} & \multirow{3}{*}{3} & \multirow{3}{*}{4} & \multicolumn{2}{|c|}{$\overline{\mathrm{KM}}$} & 18 & 9 & 8 & 14 & \multicolumn{2}{|c|}{17} \\
\hline & & & \multicolumn{2}{|c|}{$\overline{\mathrm{CM}}$} & 84 & 15 & 8 & $\overline{13}$ & \multicolumn{2}{|c|}{17} \\
\hline & & & \multicolumn{2}{|c|}{$\mathrm{PM}$} & $\mathrm{NC}$ & $\mathrm{CN}$ & $\mathrm{NC}$ & 14 & \multicolumn{2}{|c|}{17} \\
\hline & \multirow{3}{*}{0.5} & \multirow{3}{*}{2} & \multicolumn{2}{|c|}{ KM } & 11 & 7 & 8 & 7 & \multicolumn{2}{|r|}{7} \\
\hline & & & \multicolumn{2}{|c|}{$\mathrm{CM}$} & 22 & 8 & 8 & 7 & & 7 \\
\hline & & & & $\bar{M}$ & $\mathrm{NC}$ & $\mathrm{NC}$ & 7 & 7 & & 7 \\
\hline & & & & $\mathrm{M}$ & 15 & 8 & 7 & 9 & & 9 \\
\hline & 2 & 3 & & $\bar{M}$ & 49 & 11 & 8 & 9 & & 9 \\
\hline & & & & $\mathrm{M}$ & $\mathrm{NC}$ & $\mathrm{NC}$ & 8 & 9 & & 9 \\
\hline Function & & $x_{0}$ & $x_{1}$ & Metl & & & & $\overline{\alpha \alpha}$ & & \\
\hline Function & & $x_{0}$ & $x_{1}$ & Metr & & 10 & 1 & 0.1 & 0.01 & 0.001 \\
\hline & & & & $\overline{\mathrm{KI}}$ & & 8 & 6 & 6 & 6 & 6 \\
\hline & & 2 & 3 & $\overline{\mathrm{Cl}}$ & & 8 & 6 & 6 & 6 & 6 \\
\hline & & & & $\overline{\mathrm{PI}}$ & & 8 & 6 & 6 & 6 & 6 \\
\hline & & & & $\overline{\mathrm{KI}}$ & & 7 & 6 & 6 & 6 & 6 \\
\hline$x^{3}-2 x-5$ & & -3 & 2 & $\mathrm{Cl}$ & & 7 & 6 & 6 & 6 & 6 \\
\hline & & & & $\overline{\mathrm{PI}}$ & & 7 & 6 & 6 & 6 & 6 \\
\hline & & & & $\overline{\mathrm{KI}}$ & & 8 & 9 & 10 & 10 & 10 \\
\hline & & 1 & 4 & $\overline{\mathrm{Cl}}$ & & 9 & 9 & 10 & 10 & 10 \\
\hline & & & & $\overline{\mathrm{PI}}$ & & 12 & 9 & 10 & 10 & 10 \\
\hline & & & & $\overline{\mathrm{KI}}$ & & 11 & 9 & 8 & 8 & 8 \\
\hline & & 1 & 2 & $\mathrm{Cl}$ & & 11 & 9 & 8 & 8 & 8 \\
\hline & & & & $\overline{\mathrm{PI}}$ & & 13 & 9 & 8 & 8 & 8 \\
\hline & & & & $\overline{\mathrm{KI}}$ & & 11 & 14 & $\mathrm{NC}$ & $\mathrm{NC}$ & $\mathrm{NC}$ \\
\hline $\mathbf{x} \exp (\mathbf{x})-1$ & & -1 & 3 & $\overline{\mathrm{Cl}}$ & & 13 & $\mathrm{NC}$ & $\mathrm{NC}$ & $\mathrm{NC}$ & $\mathrm{NC}$ \\
\hline & & & & $\overline{\mathrm{PI}}$ & & 17 & 9 & $\mathrm{NC}$ & $\mathrm{NC}$ & $\mathrm{NC}$ \\
\hline & & & & $\overline{\mathrm{Kl}}$ & & 7 & 9 & 15 & 17 & 18 \\
\hline & & -3 & 1 & $\overline{\mathrm{Cl}}$ & & 9 & 7 & 13 & 17 & 18 \\
\hline & & & & $\overline{\mathrm{PI}}$ & & $\mathrm{NC}$ & 10 & 11 & 17 & 18 \\
\hline & & & & $\overline{\mathrm{Kl}}$ & & 12 & 11 & 11 & 11 & 11 \\
\hline & & 2 & 3 & $\overline{\mathrm{Cl}}$ & & 13 & 11 & 11 & 11 & 11 \\
\hline & & & & $\overline{\mathrm{PI}}$ & & 13 & 11 & 11 & 11 & 11 \\
\hline & & & & $\mathrm{KI}$ & & 10 & 7 & 9 & 9 & 9 \\
\hline $\mathbf{x} \exp (\mathbf{x})-\cos$ & & 0 & 2 & $\overline{\mathrm{Cl}}$ & & 11 & 7 & 9 & 9 & 9 \\
\hline & & & & $\mathrm{PI}$ & & 15 & 7 & 9 & 9 & 9 \\
\hline & & & & $\mathrm{KI}$ & & 7 & 8 & 11 & 11 & 11 \\
\hline & & -1 & 1 & $\overline{\mathrm{Cl}}$ & & 9 & 8 & 11 & 11 & 11 \\
\hline & & & & $\overline{\mathrm{PI}}$ & & 12 & 8 & 10 & 11 & 11 \\
\hline
\end{tabular}




\section{References}

[1] I. K. Argyros, On a class of secant-like methods for solving nonlinear equations, Numer. Algor., 54, No. 4 (2010), 485-501.

[2] I. K. Argyros and S. Hilout, Secant-type methods and non-discrete induction, Numer. Algor., 61, No. 3 (2012), 397-412.

[3] K. E. Atkinson, An Introduction to Numerical Analysis, Second Edition. John Wiley \& Son Inc., New York, 1989..

[4] L. Chen, A family of improved secant-like method with super-linear convergence, International Journal of Mathematical, Computational, Physical and Quantum Engineering, 7 , No. 4 (2013), 375-378..

[5] J.A. Ezquerro, M.A. Hernndez, N. Romero and A.I. Velasco, Improving the domain of starting points for secant-like methods, Applied Mathematics and Computation, 219, No. 8 (2012) 3677-3692.

[6] J.A. Ezquerro, A. Grau, M. Grau-Snchez, M.A. Hernndez, and M. Noguera, Analysing the efficiency of some modifications of the secant method, Computers and Mathematics with Applications, 64, No. 6 (2012), 2066-2073..

[7] J.A. Ezquerro, M. Grau-Snchez, M.A. Hernndez and M. Noguera, Semi-local convergence of secant-like methods for differentiable and nondifferentiable operator equations, J. Math. Anal. Appl., 398,No. 1 (2013), 100-112.

[8] M.A. Hernandez, M.J. Rubio and J.A. Ezquerro, Solving a special case of conservative problems by Secant-like methods, Applied Mathematics and Computation, 169, No. 2 (2005), 926-942.

[9] Z. Hui, L. De-Sheng and L. Yu-Zhong, A new method of secant-like for nonlinear equations, Commun. Nonlinear Sci. Numer. Simulat., 14, No. 7 (2009), 2923-2927.

[10] V. Kanwar, J.R. Sharma amd Mamta, A new family of Secant-like method with superlinear convergence, Applied Mathematics and Computation, 171, No. 1 (2005), 104-107..

[11] H. Ren, An improved convergence theorem for a class of Secant-like methods, Applied Mathematics and Computation, 189, No. 1 (2007), 472-481,.

[12] H. Ren, A second-derivative-free modified Secant-like method with order $2.732 \ldots$ for unconstrained optimization, Applied Mathematics and Computation, 202, No. 2 (2008) 688-692.

[13] H. Ren, Q. Wu and W. Bi, On convergence of a new secant-like method for solving nonlinear equations, Applied Mathematics and Computation, 217, No. 2 (2010), 583-589.

[14] X. Wang, J. Kou and C. Gu, A new modified secant-like method for solving nonlinear equations, Computers and Mathematics with Applications, 60, No. 6 (2010), 1633-1638. 
